\title{
Comparisons on Efficacy of Elcatonin and Limaprost Alfadex in Patients with Lumbar Spinal Stenosis and Concurrent Osteoporosis: A Preliminary Study Using a Crossover Design
}

\author{
Tsukasa Kanchiku ${ }^{1}$, Yasuaki Imajo ${ }^{1}$, Hidenori Suzuki1, Yuichiro Yoshida ${ }^{1}$, \\ Toshihiko Taguchi ${ }^{1}$, Toshikatsu Tominaga ${ }^{2}$, Koichiro Toyoda ${ }^{3}$ \\ ${ }^{1}$ Department of Orthopedic Surgery, Yamaguchi University Graduate School of Medicine, Yamaguchi, Janpan \\ ${ }^{2}$ Department of Orthopedic Surgery, Yamaguchi Rosai Hospital, Yamaguchi, Janpan \\ ${ }^{3}$ Department of Orthopedic Surgery, Yamaguchi Grand Medical Center, Yamaguchi, Janpan
}

\begin{abstract}
Study Design: Multicenter prospective study with a crossover design.
Purpose: The objective of this study is to compare the efficacy of limaprost alfadex (LP) and elcatonin (EL) for lumbar spinal stenosis (LSS) patients with concurrent osteoporosis.

Overview of Literature: It has been increasingly important to improve quality of life by establishing appropriate conservative treatments for LSS patients with concurrent osteoporosis who will presumably continue to increase due to the percentage of the aging elevations, however there is no prospective study.

Methods: A total of 19 patients with LSS and concurrent osteoporosis were enrolled in this study. The patients were divided into two groups and compared using a crossover design. The Japanese Orthopaedic Association Back Pain Evaluation Questionnaire (JOABPE0) and short-form (SF)-8 health survey scale were used for clinical evaluations.

Results: There was a significant improvement of buttock-leg pain and numbness in the EL group. A significant improvement of impaired walking function was noted for the LP group according to the JOABPEQ while the rest of the items in the JOABPEQ showed no significant differences. The SF-8 health survey revealed that somatic pains and physical summary scores in the EL group and physical functioning and physical summary scores in the LP group tended to improve but not to any statistically significant extents.

Conclusions: Concomitant uses of EL may be useful in patients who do not respond satisfactorily to the treatments of LP for 6-8 weeks.
\end{abstract}

Keywords: Lumbar spinal stenosis; Osteoporosis; Pharmacotherapy; Limaprost alfadex; Elcatonin

\section{Introduction}

With the aging of the population, cases of spinal degen- erative diseases have been increasing; especially cases of lumbar degenerative disease are frequently encountered in the daily orthopedic practice settings. Lumbar spi-

Received Apr 29, 2013; Revised May 18, 2013; Accepted May 21, 2013

Corresponding author: Tsukasa Kanchiku

Department of Orthopedic Surgery, Yamaguchi University Graduate School of Medicine,

1-1-1 Minami-kogushi, Ube, Yamaguchi 755-8505, Japan

Tel: +836-22-2268, Fax: +836-22-2267, E-mail: tkanchik@yamaguchi-u.ac.jp 
nal stenosis (LSS) is generally recognized as a disorder characterized by low back pains, leg pains, numbness of the lower extremities, and also intermittent claudication, wherein the cauda equine and nerve roots are entrapped due to narrowing of the spinal canal and/or the foramina nervosa, causing blood circulatory impediment and a consequent anoxic condition to produce a variety of symptoms. Treatment of LSS with mild to moderate severity consists primarily of pharmacotherapy with such agents as nonsteroidal anti-inflammatory drugs (NSAIDs) and prostaglandin $\left(\mathrm{PGE}_{1}\right)$, combined with conservative measures such as physical therapy, orthotic appliance therapy and lifestyle guidance. Surgery is considered if these conservative treatments which have failed to improve leg pains, numbness of the lower extremities and dysbasia if there were any vesicorectal disorders. Patients are often the elderly; therefore, a number of patients with high risk of complications are reluctant to undergo surgical treatments. Conservative treatment is being continued for such patients.

Limaprost alfadex is primarily used for improvement of nerve blood flows via its peripheral vasodilatatory action, and it is currently positioned as the first-line pharmacotherapy for LSS. However, the clinical improvement rate for the therapy is approximately $50 \%$ [1], and a sufficient clinical effect has not yet been obtained.

The number of patients with osteoporosis has been increasing consistently with the aging of the population and is estimated to be 12.8 million, and the principal clinical manifestations of the diseases are fractures arising from fragility of bones and associated disabilities and chronic low back pains. Calcitonin (elcatonin) is an inhibitor of bone resorption with a central analgesic effect [2] and is one of the first-line treatments for painful high-turnover osteoporosis. It is reported to have a blood flow-improving effect on peripheral blood vessels [3] and is suggested to show efficacy for LSS [4], but no sufficient evidences has been obtained.

Attempts to improve quality of life by establishing appropriate conservative treatments for LSS patients with concurrent osteoporosis will presumably continue to increase due to the elevating percentages of the aged which lead to reductions in medical expenses (currently a major problem in Japan). The purpose of this study is to compare the efficacy of limaprost alfadex and elcatonin for LSS with concurrent osteoporosis in the same patient when using a crossover design.

\section{Materials and Methods}

The study included patients aged 50 years and older who were newly diagnosed as having LSS and concurrent primary osteoporosis at our hospital or affiliated medical institutions, and are capable of unaided walking. All subjects were given written informed consents in advance to participate in this study.

Exclusion criteria were as follows: severe LSS indicated for surgery, symptoms due to chronic arterial occlusive diseases, any intercurrent serious disorders, dementia, errors in assumptions due to serious neurological disorders, a history of hypersensitivity to elcatonin and limaprost alfadex; thus, such patients were judged to be inadequate for subjects of this study. The present study was conducted with the approval of the institutional review board. Using the Clinical Diagnosis Support Tool to Identify Patients with LSS of the Japanese Society of Spine Surgery and Related Research [5], the LSS was diagnosed when the patient scored 7 points or more. The Diagnostic Criteria for Primary Osteoporosis (revised in 2000) were used for the diagnosis of osteoporosis, whereby patients meeting these criteria were diagnosed as having primary osteoporosis.

The patients with LSS and concurrent osteoporosis were divided into the following two groups and compared by using a crossover design: in one (EL) group, elcatonin (Elcitonin Injection 20S) was intramuscularly administered once weekly for 4 weeks, followed by a 1-week washout period and then limaprost alfadex (LP; Opalmon or Prorenal) was administered orally in a daily dose of $15 \mu \mathrm{g}$ (given in 3 divided doses) for 4 weeks; whereas in the other (LP) group, limaprost alfadex was administered orally as stated above for 4 weeks, followed by a 1-week washout period, and then elcatonin was given as above for 4 weeks. Patients were randomized to the treatment groups by the secretariat using the minimization method as to minimize differences in distribution of the pertinent factors between the two groups.

Drugs for concomitant therapies which might affect the efficacy evaluation of the study (analgesics, muscle relaxants, corsets, etc.) were prohibited. Nerve block such as the epidural block was also excluded. Patient demographic characteristics included gender, age, body weight, duration of illness, complications, medical history, and disease type; and each patient was evaluated using the Japanese Orthopaedic Association Back Pain Evaluation 
Table 1. Patient demographic characteristics

\begin{tabular}{|c|c|c|c|c|}
\hline Characteristic & Overall & EL group & LP group & $\begin{array}{c}p \text {-value } \\
\text { (EL vs. LP) }\end{array}$ \\
\hline No. of patients enrolled & 19 & 9 & 10 & - \\
\hline Efficacy analysis set & 15 & 9 & 6 & - \\
\hline Patients who discontinued treatment & 4 & 0 & 4 (lost to follow-up) & - \\
\hline Age (yr) & $71.1 \pm 8.6(55-86)$ & $69.9 \pm 9.6(55-86)$ & $70.8 \pm 2.6(67-74)$ & 0.82 \\
\hline Gender (male:female) & 5:10 & $3: 6$ & $2: 4$ & 1 \\
\hline Duration of illness (mo) & $17.9 \pm 14.5(2-61)$ & $13.3 \pm 6.7(2-24)$ & $31.3 \pm 21.3(14-61)$ & 0.04 \\
\hline Support tool (score) & $12.2 \pm 3.0(7-16)$ & $12.1 \pm 2.6(8-15)$ & $13.5 \pm 2.2(10-16)$ & 0.3 \\
\hline Disease type & & & & - \\
\hline Nerve root type & 10 & 4 & 2 & \\
\hline Cauda equine type & 6 & 4 & 2 & \\
\hline Mixed type & 2 & 1 & 1 & \\
\hline Unknown & 1 & 0 & 1 & \\
\hline
\end{tabular}

Values are presented as number or mean \pm standard deviation (range).

EL group, received elcatonin 20 U/week for 4 weeks; LP group, received limaprost alfadex $15 \mathrm{mg} /$ day for 4 weeks.

Questionnaire (JOABPEQ) and short-form (SF)-8 health survey scale. Assessments were performed at randomization, and at 4, 5, and 9 weeks after randomization. Treatment responses were evaluated by comparing the primary variables between the two treatment groups. Statistical analysis was performed by using SPSS ver. 19.0 (IBM Co., Armonk, NY, USA) and paired $t$-test for intergroup comparisons. Any intergroup differences found were considered statistically significant if the level of significance was less than $5 \%$.

\section{Results}

A total of 19 patients were enrolled in this study, 4 patients of the LP group dropped out (lost due to follow up after washouts); therefore, the effective analysis set comprised of 15 patients ( 9 in the EL group and 6 in the LP group). Of the patient demographic characteristics assessed, only the duration of illness was significantly longer for the LP group while none of the rest showed any significant differences between the groups (Table 1).

When comparing clinical responses for 4 weeks of treatment with the baseline, there was no significant difference in visual analogue scores (VAS) score for low back pains in either groups, but there was a significant improvement of buttock-leg pains and numbness in the EL group (Figs. 1-3). A significant improvement of impaired walking function was noted for the LP group according to the JOABPEQ while the rest of the items in the JOABPEQ showed no significant differences from the baseline or between the two treatment groups (Figs. $4-8)$. The SF-8 health survey revealed that somatic pains and physical summary scores in the EL group and physical functioning and physical summary scores in the LP group tended to improve but not to any statistically sig-

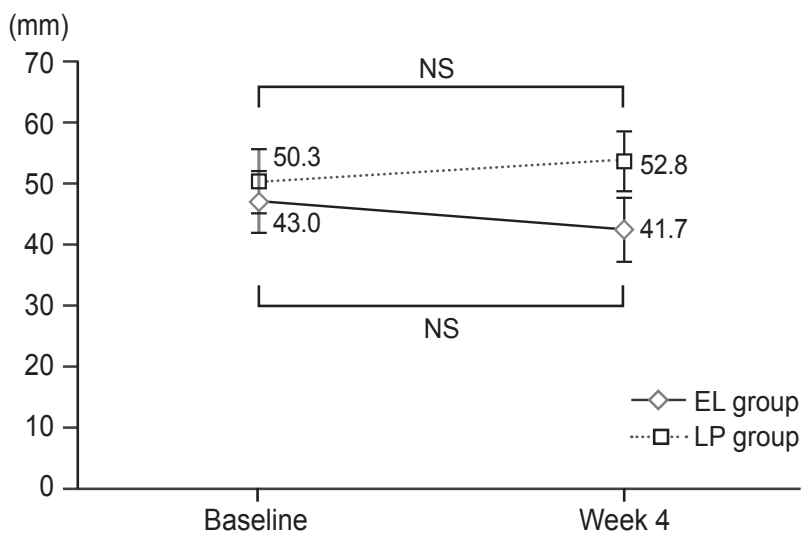

Fig. 1. Low back pain visual analogue scores (VAS) scores over time. VAS scores for low back pain were assessed at baseline and at week 4 of treatment. The results are expressed as mean \pm standard deviation. No significant changes were noted at week 4 of treatment in either group as compared to baseline scores; nor were there any significant differences between these two groups. NS, not significant (paired $t$ test); EL, elcatonin; LP, limaprost alfadex. 
nificant extents (Figs. 9, 10).

\section{Discussion}

This study is considered to be the first multicenter prospective crossover clinical study to investigate the effects

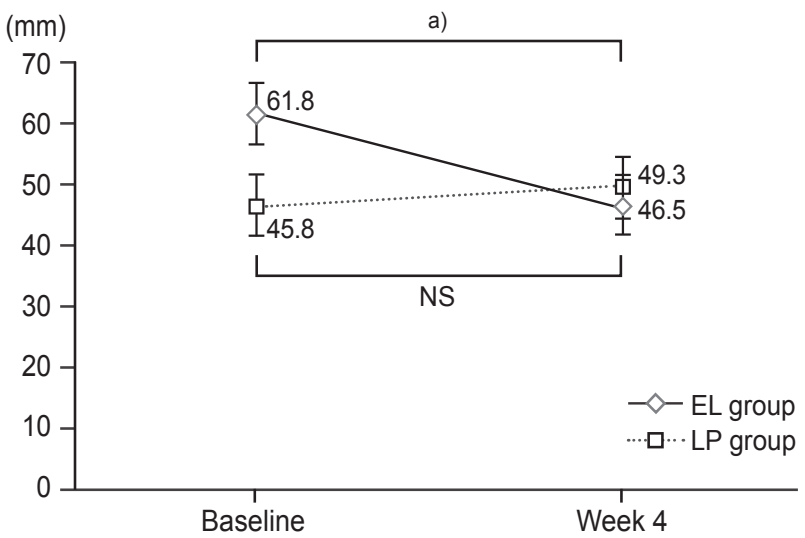

Fig. 2. Buttock-leg pain visual analogue scores (VAS) scores over time. VAS scores for buttock-leg pain were assessed at baseline and at week 4 of treatment. The results are expressed as mean \pm standard deviation. The EL group exhibited a significant improvement of buttockleg pain at week 4 of treatment as compared with baseline scores; whereas the LP group showed no significant changes. However, no significant differences were observed between these two groups. EL, elcatonin; LP, limaprost alfadex. ${ }^{\text {al }} p<0.05$ Significant differences by paired $t$-test.

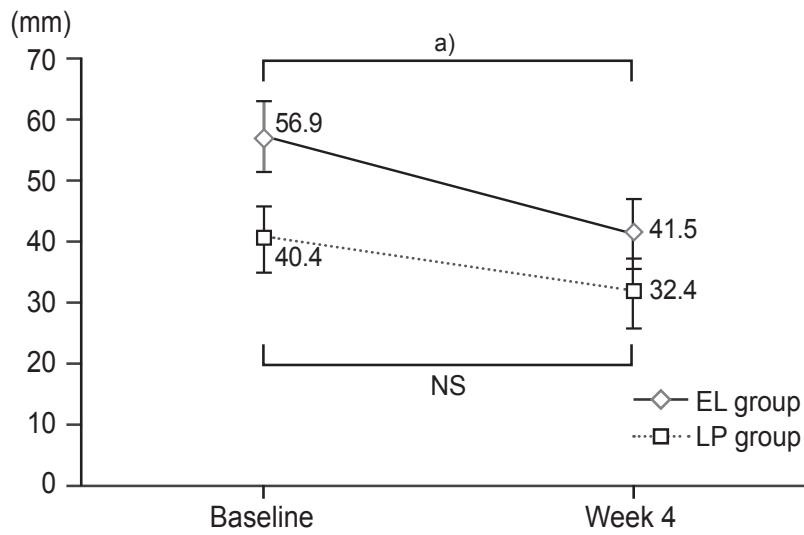

Fig. 3. Buttock-leg numbness visual analogue scores (VAS) scores over time. VAS scores for buttock-leg numbness were assessed at baseline and at week 4 of treatment. The results are expressed as mean \pm standard deviation. Buttock-leg numbness significantly improved in the EL group at week 4 of treatment as compared with baseline scores; whereas in the LP group, no significant changes were observed. However, there were no significant differences between these two treated groups. NS, not significant; EL, elcatonin; LP, limaprost alfadex. ${ }^{\text {a) }} p<0.05$. Significant differences by paired $t$-test. of elcatonin and limaprost alfadex in patients with newly diagnosed LSS associated with osteoporosis. Conventional pharmacotherapy for LSS includes NSAIDs, muscle relaxants and methylcobalamin, but no sufficient evidences were gained regarding the efficacy of these drugs.

Limaprost alfadex has been demonstrated to be effec-

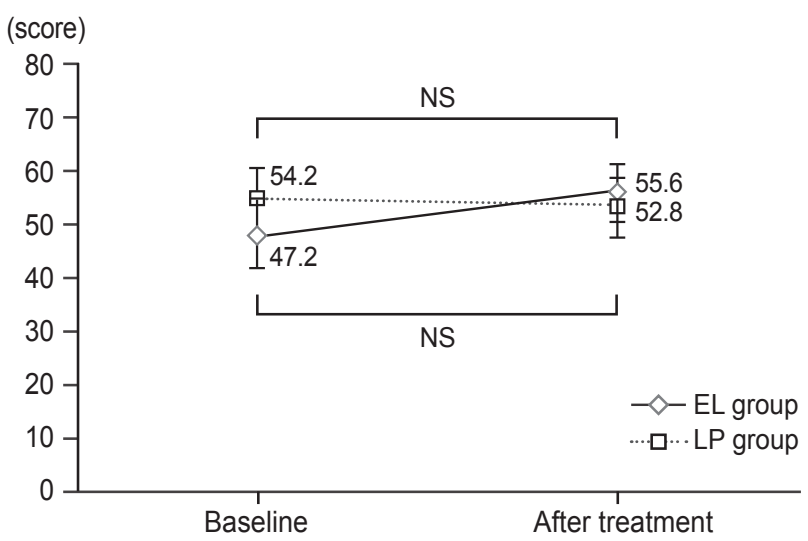

Fig. 4. Changes in pain-related disorders over time based on JOABPEO data. Pain-related disorder scores were assessed at baseline and at week 4 of treatment. The results are expressed as mean \pm standard deviation. Neither group displayed any significant changes from baseline at week 4 of treatment. Nor were there any differences between these two groups. JOABPEQ, Japanese Orthopaedic Association Back Pain Evaluation Questionnaire; NS, not significan (paired $t$-test); EL, elcatonin; LP, limaprost alfadex.

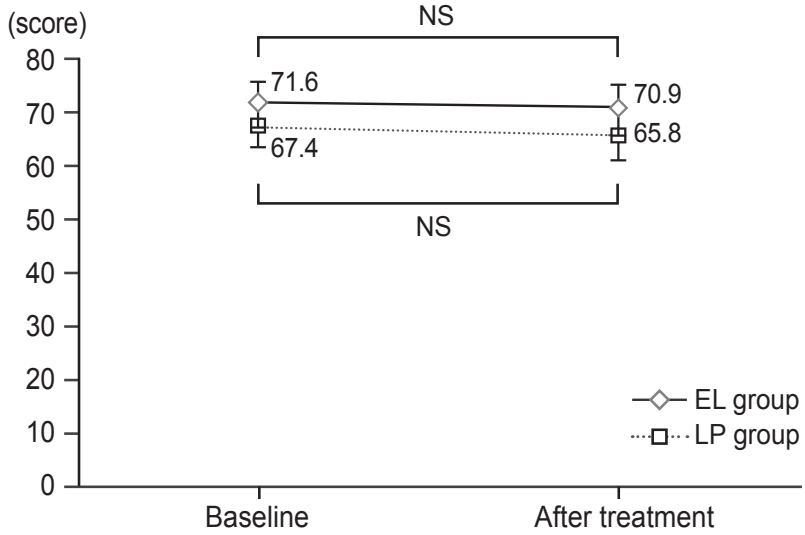

Fig. 5. Changes in low back pain functioning impairment scores over time based on JOABPEO data. Low back pain function impairment scores were assessed at baseline and at week 4 of treatment. The results are expressed as mean \pm standard deviation. Neither group displayed any significant changes from baseline at week 4 of treatment. Nor were there any differences between these two groups. JOABPEO, Japanese Orthopaedic Association Back Pain Evaluation Questionnaire; NS, not significan (paired $t$-test); EL, elcatonin; LP, limaprost alfadex. 
tive over a short period of time for patients with LSS presenting neurogenic claudication and cauda equine symptoms associated with numbness in the lower extremities [6]. In the present study, the efficacy of limaprost alfadex was also noted albeit for a short period of 4 weeks, as indicated by a significant improvement of the impaired

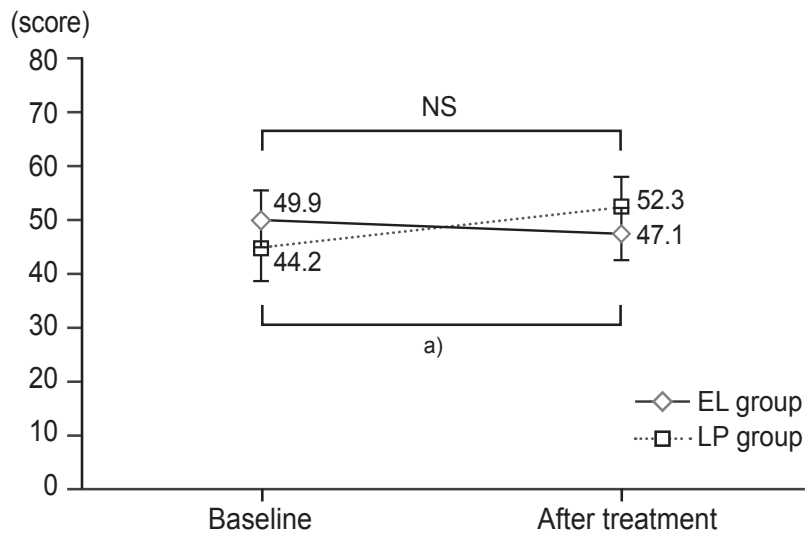

Fig. 6. Changes in walking function impairment factors over time based on JOABPEO data. Walking function impairment scores were assessed at baseline and at week 4 of treatment. The results are expressed as mean \pm standard deviation. Impaired walking function significantly improved from baseline at week 4 of treatment in the LP group, while no significant changes were noted in the EL group. No difference was noted between the two groups, however. JOABPEO, Japanese Orthopaedic Association Back Pain Evaluation Questionnaire; NS, not significan; EL, elcatonin; LP, limaprost alfadex. ${ }^{a)} p<0.05$. Significant differences by paired $t$-test.

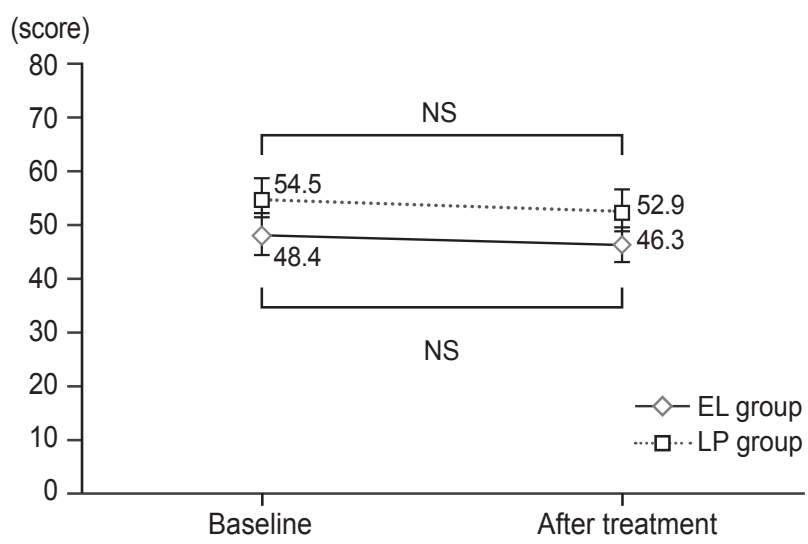

Fig. 7. Changes in social living impairment factors over time based on Japanese Orthopaedic Association Back Pain Evaluation Questionnaire data. Social living impairment scores were assessed at baseline and at week 4 of treatment. The results are expressed as mean \pm standard deviation. Neither group displayed any significant changes from baseline at week 4 of treatment. No differences were noted between the two groups. JOABPEQ, Japanese Orthopaedic Association Back Pain Evaluation Questionnaire; NS, not significan (paired $t$-test); EL, elcatonin; LP, limaprost alfadex. walking function score in the JOABPEQ (Fig. 6); and a tendency for improvements of physical functioning and physical component score (physical summary score) in the SF-8 health survey (Fig. 10) was noted in the group receiving the drug. There have been sporadic reports demonstrating the efficacy of elcatonin for intermittent

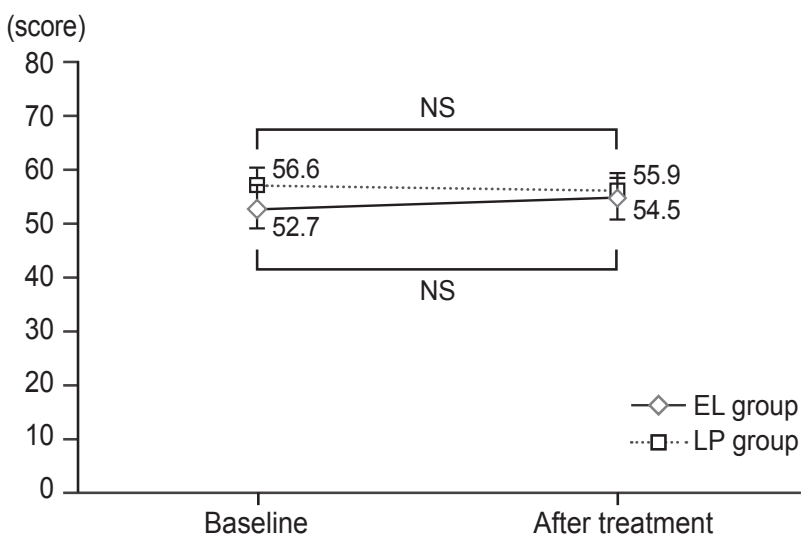

Fig. 8. Changes in psychological impairment factors over time based on Japanese Orthopaedic Association Back Pain Evaluation Questionnaire data. Psychological impairment scores were assessed at baseline and at week 4 of treatment. The results are expressed as mean \pm standard deviation. Neither group displayed any significant changes from baseline at week 4 of study treatment. Nor were there any differences between these two groups. NS, not significan (paired $t$-test); EL, elcatonin; LP, limaprost alfadex.

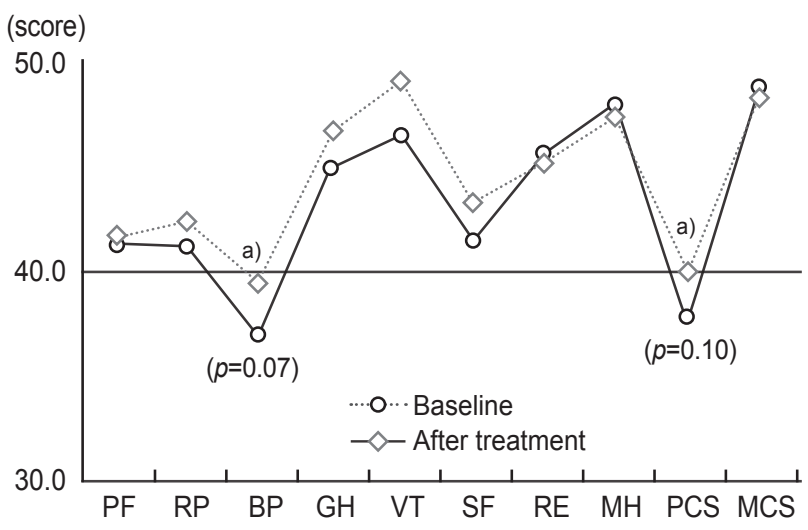

Fig. 9. Changes in SF-8 survey subscale scores and summary scores over time (elcatonin group). SF-8 eight subscale scores and summary scores were assessed at baseline and at week 4 of treatment. The results are expressed in mean scores. At week 4 of treatment, BP and PCS tended to improve with no significant differences as compared with baseline scores. Eight subscales were obtained: PF, RP, BP, GH, VT, $\mathrm{SF}, \mathrm{RE}$, and $\mathrm{MH}$. These scores are further summarized into two overall scores: PCS and MCS. SF-8, short-form-8; PF, physical functioning; $\mathrm{RP}$, role physical; $\mathrm{BP}$, bodily pain; $\mathrm{GH}$, general health; VT, vitality; SF, social functioning; RE, role emotional; $\mathrm{MH}$, mental health; PCS, physical component summary; MCS, mental component summary. ${ }^{a} p$-value (paired $t$-test). 
claudication, including the first one by Porter and Miller [4], showing the efficacy of the drugs for conditions due to LSS. The present study has failed to show any significant improvements of dysbasia score in the elcatonin treated group. However, buttock-leg pains and numbness were significantly improved in terms of VAS score following the treatment, as compared to the baseline (Figs. $2,3)$. In the SF-8 health survey, there was a tendency for improvements on somatic pain and physical component score (Fig. 9). Wakioka et al. [7] reported a significant improvement of impaired walking function occurring after 12 weeks of elcatonin treatment. A longer continued treatment period may be necessary in order to achieve significant improvements on the walking function score since the treatment duration in the present study was only for 4 weeks. In the nerve root type LSS, pain symptoms are prominent and dysbasia may be milder as compared with cauda equine type and mixed type, thus, the analgesic effects may hardly be paralleled to the improvements of walking ability. On the other hand, significantly improved buttock-leg pain and numbness was attained from an early stage of treatment, as shown by previous reports, thus, providing evidence in support of its efficacy. Ito et al. [8] reported that the level of high-threshold $\mathrm{Na}$ channel expression is augmented by following treatment with elcatonin at the $\mathrm{C}$-afferent terminals, being nociceptive

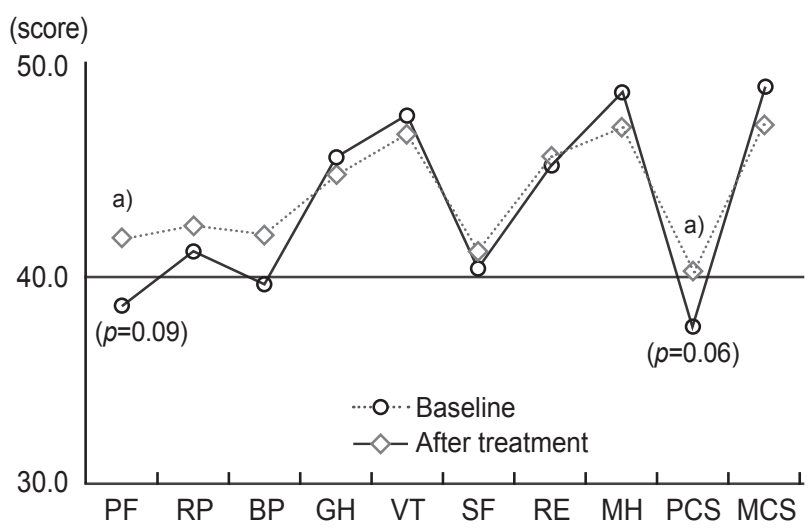

Fig. 10. Changes in SF-8 survey subscale scores and summary scores over time (limaprost alfadex group). SF-8 eight subscale scores and summary scores were assessed at baseline and at week 4 of treatment. The results are expressed in mean scores. At week 4 of study treatment, the PF and PCS tended to improve with no significant differences as compared with baseline scores. SF-8, short-form-8; PF, physical functioning; $\mathrm{RP}$, role physical; $\mathrm{BP}$, bodily pain; $\mathrm{GH}$, general health; VT, vitality; SF, social functioning; RE, role emotional; $\mathrm{MH}$, mental health; PCS, physical component summary; MCS, mental component summary. ${ }^{\text {a) }} p$-value (paired $t$-test). neurons in the posterior horn of the spinal cord, in ovariectomy-induced hyperalgesia rats, indicating that recovery of the descending inhibitory system also contributes to development of the analgesic effects. Furthermore, an analgesic effect against neurogenic pain was observed in chronic constriction injury (CCI) model rats [9]. Elcatonin was demonstrated to exert its blood flow to improve effects on the affected leg with decreased blood flows but not on the intact leg in CCI model rats [10]. Nagai [11], on the other hand, reported that when following treatment with elcatonin, the blood flow improvement rate was higher in regions with diminished blood flow than in regions with normal blood flow in patients with osteoporosis. Intraspinal-endoscopically, the existence of a relative hypoxic state due to arterial ischemia and venous congestion evoked by elevated extradural pressure is considered to account for the genesis of neurogenic intermittent claudication. Ogata et al. [12] considered that the effects of elcatonin on the improvement of such symptoms might be attributed to the improvement of an oligemic/ ischemic state via its blood flow-improving effects on the blood vessels compressed due to spinal canal stenosis. Like $\mathrm{PGE}_{1}$, elcatonin may facilitate improvements of neurotransmission velocity by increasing cauda equina blood flows. The effects of elcatonin on improving the $\mathrm{Na}$ channel-mediated peripheral nerve excitation threshold [9] and that through the descending serotonergic nerve system [8] are considered to have beneficial effects upon the cauda equine and nerve roots. In addition, as seen from the improvement of somatic pain and physical component score in the SF-8 health survey, the psychological effects produced by weekly injections and clinic interviews may support the symptom-ameliorating effects of elcatonin. Minimal materials and short follow-up periods were the limitations for this study. Further studies are necessary for validation of these results.

\section{Conclusions}

Concomitant use of elcatonin may be useful in patients who do not respond satisfactorily to the treatment of limaprost alfadex for 6 to 8 weeks.

\section{Conflict of Interest}

No potential conflict of interest relevant to this article was reported. 


\section{References}

1. Kurihara A, Kataoka O, Sugawara S, Sano S, Shirai Y. Clinical benefit on OP-1206 a-CD on lumbar spinal canal stenosis. Multi-center comparative doubleblind clinical study. Rinsho Iyaku 1996;12:511-29.

2. Knopp JA, Diner BM, Blitz M, Lyritis GP, Rowe BH. Calcitonin for treating acute pain of osteoporotic vertebral compression fractures: a systematic review of randomized, controlled trials. Osteoporos Int 2005;16:1281-90.

3. Nagai T, Sakamoto K, Miyaoka H. Three cases in which a calcitonin(elcatonin) improved blood flow of the legs. J Eas Japan Assoc Orthop Trauma 2006;18:493-7

4. Porter RW, Miller CG. Neurogenic claudication and root claudication treated with calcitonin. A doubleblind trial. Spine (Phila Pa 1976) 1988;13:1061-4.

5. Konno S, Hayashino Y, Fukuhara S, et al. Development of a clinical diagnosis support tool to identify patients with lumbar spinal stenosis. Eur Spine J 2007;16:1951-7.

6. Matsudaira K, Seichi A, Kunogi J, et al. The efficacy of prostaglandin E1 derivative in patients with lumbar spinal stenosis. Spine (Phila Pa 1976) 2009;34:115-20.
7. Wakioka T, Sato K, Mitsukawa M, et al. The efficacy of elcatonin in the treatment of lumbar spinal canal stenosis complicated by osteoporosis. J New Remedies Clinic 2011:2087-95.

8. Ito A, Kumamoto E, Takeda M, Shibata K, Sagai H, Yoshimura M. Mechanisms for ovariectomy-induced hyperalgesia and its relief by calcitonin: participation of 5-HT1A-like receptor on C-afferent terminals in substantia gelatinosa of the rat spinal cord. J Neurosci 2000;20:6302-8.

9. Ito A, Takeda M, Yoshimura T, et al. Anti-hyperalgesic effects of calcitonin on neuropathic pain interacting with its peripheral receptors. Mol Pain 2012;8:42.

10. Yoshimura T, Ito A, Saito SY, Takeda M, Kuriyama $\mathrm{H}$, Ishikawa T. Calcitonin ameliorates enhanced arterial contractility after chronic constriction injury of the sciatic nerve in rats. Fundam Clin Pharmacol 2012;26:315-21.

11. Nagai T, Sakamoto K, Miyaoka H. The effect of calcitonin (elcatonin) on blood flow. Showa Igakkai Zasshi 2007;67:469-78.

12. Ogata T, Mutaguchi K, Ohki M, et al. The effectiveness of elcatonin in lumbar spinal canal stenosis patients with osteoporosis: multicenter trial. Ther Res 2009;30:997-1004. 